\section{Milieu Intérieur : définir les limites d'une réponse immunitaire saine pour mieux comprendre la maladie}

Darragh Duffy ${ }^{1,2}$

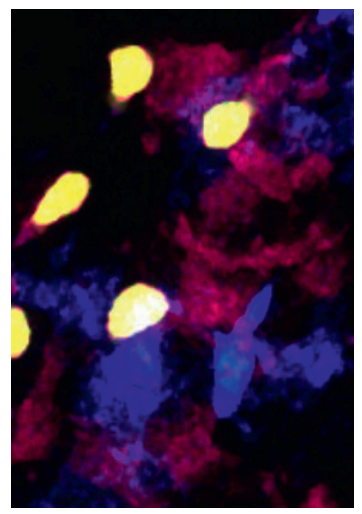

${ }^{1}$ Immunobiologie des cellules dendritiques, Institut Pasteur, 25 , rue du Docteur Roux, 75015 Paris, France. ${ }^{2}$ Inserm U1223, 25, rue du Docteur Roux, 75015 Paris, France. darragh.duffy@pasteur.fr
Le consortium LabEx Milieu Intérieur a été créé en $2011^{1}$ pour mieux définir ces différences immunitaires dans une population en bonne santé et pour identifier leurs déterminants génétiques et environnementaux. Une telle compréhension est cruciale pour accomplir la promesse d'une médecine de précision qui pourra tenir compte de la spécificité de la réponse immunitaire d'un individu et ainsi s'assurer que les stratégies de santé publique tirent profit des progrès scientifiques récents.

\section{Le besoin de comprendre la variabilité de la réponse immunitaire}

Le système immunitaire est responsable de la préservation d'un état sain, de la prévention des infections et du maintien de l'homéostasie. Chez certaines personnes, cependant, un dysfonctionnement immunitaire peut survenir et entraîner une susceptibilité accrue aux infections, à l'inflammation, à l'auto-immunité, aux allergies ou même au cancer. Une telle hétérogénéité

Vignette (Photo @ Inserm - Sebastian Amigorena)

${ }^{1}$ http://www.milieuinterieur.fr/en individuelle dans la réponse immunitaire peut également avoir un impact majeur sur la probabilité de réponse au traitement ou sur le développement d'effets secondaires à l'administration d'un vaccin. La plupart des études visant à comprendre dans quelle mesure la variation des réponses immunitaires est associée à l'immunopathologie sensu lato ont adopté une approche fondée sur la maladie, ce qui a permis d'obtenir une compréhension approfondie des mécanismes immunitaires. Néanmoins, pour utiliser cette information dans le diagnostic et la prise en charge de la maladie, il est nécessaire de définir les paramètres de base de la fonction immunitaire dans l'ensemble de la population humaine «saine». Afin d'atteindre cet objectif, le projet Milieu Intérieur vise à fournir une base pour définir les perturbations dans les réponses immunitaires d'un individu.

Les réponses immunitaires humaines présentent des niveaux élevés de variabilité interindividuelle dans toutes les populations [1]. Une partie de cette variabilité résulte de la nature souple et dynamique des réponses immunitaires qui rend possible des stratégies de vaccination et thérapeutiques pour une variété de maladies allant des maladies infectieuses au cancer. Cependant, cette variabilité inhérente peut aussi représenter des défis, car la susceptibilité aux infections, la gravité de la maladie et la réponse aux traitements médicaux varient énormément d'une personne à l'autre. Les pratiques médicales et les politiques de santé publique adoptent généralement un modèle «taille unique » pour la gestion des maladies et la mise au point de médicaments. Cette approche ne tient pas compte de l'hétérogénéité des 


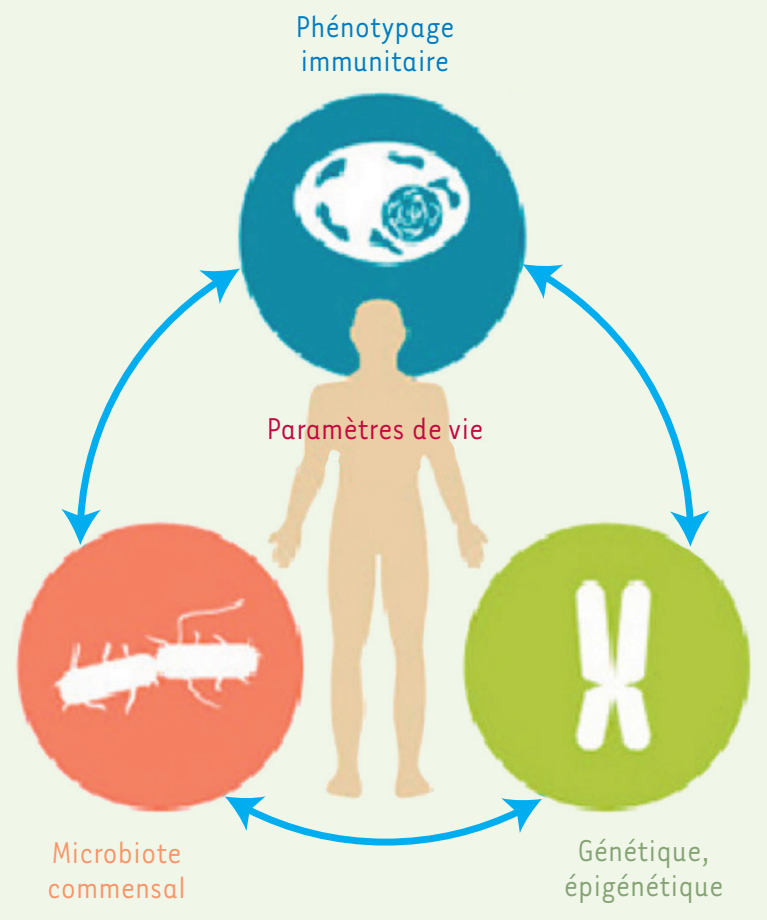

Figure 1. Intégration dans Milieu Intérieur des données de phénotypage de la réponse immunitaire, de génétique et d'épigénétique, ainsi que du microbiome, dans le but d'étudier les déterminants de la variabilité immunitaire.

réponses immunitaires individuelles qui a probablement une incidence sur la réponse au traitement, ou sur l'efficacité et l'apparition d'effets secondaires consécutifs à l'administration de vaccin ou de traitement. Parmi les exemples bien connus de vaccination, mentionnons le vaccin contre l'hépatite B pour lequel la séroconversion échoue pour 5 à $10 \%$ des individus sains [2]. Bien que les vaccins personnalisés puissent s'appliquer à des maladies comme le cancer, ils ne constituent pas à l'heure actuelle une approche réalisable pour des interventions de prévention auprès de la population. Les stratégies de vaccination de précision peuvent cependant tenir compte de différences plus générales au sein de la population afin d'améliorer l'efficacité d'un vaccin et de minimiser des effets indésirables potentiels. En raison de la complexité des réponses immunitaires au niveau individuel et dans la population, il a été néanmoins difficile, jusqu'à présent, de définir les limites d'un système immunitaire sain ainsi que les paramètres (génétiques, épigénétiques et environnementaux) qui déterminent sa variabilité naturelle. De telles évaluations exigent des échantillons de grande taille, un consensus pour définir ce qui est «sain » et des protocoles normalisés pour le recrutement des échantillons. La variabilité des réponses immunitaires peut être due à de multiples facteurs biologiques (âge, sexe), génétiques (polymorphisme [SNP, single nucleotide polymorphism], méthylation des gènes), environnementaux (infections microbiennes, latentes ou chroniques) ou liés au mode de vie (alimentation, tabagisme, historique médical). Bien que de nombreuses études aient examiné la façon dont chacune de ces composantes influe individuellement sur les réponses immunitaires, peu de travaux ont réussi à combiner ces cofacteurs dans une analyse intégrative.

C'est dans ce contexte que le consortium Milieu Intérieur a été créé avec pour but de définir les limites normales d'une réponse immunitaire saine dans une population européenne [3]. Le projet porte le nom du concept de «milieu intérieur » du physiologiste français Claude Bernard et vise à établir les déterminants d'une réponse immunitaire saine en identifiant les facteurs génétiques et environnementaux qui contribuent à l'hétérogénéité des réponses immunitaires que I'on observe. Coordonné par l'Institut Pasteur de Paris, il s'agit d'un projet pluridisciplinaire associant des scientifiques issus du monde académique (Institut Pasteur, Institut Curie, Université Paris-Diderot, Université Paris-13, Inserm, CNRS, École Polytechnique Fédérale de Lausanne [عPFL], Suisse, et Université de Lund, Suède), du monde médical (Hôpital Européen Georges Pompidou, Hôpital Cochin et Hôpital Saint Louis), et du secteur industriel (Myriad-RBM, Généthon, Institut Roche). Ce programme soutenu par le ministère de l'Enseignement Supérieur, de la Recherche et de l'Innovation dans le cadre d'un plan Investissement d'Avenir - Laboratoire d'Excellence ( $L a b \varepsilon x$ ), et géré par l'Agence nationale de la recherche, vise à fournir aux laboratoires reconnus internationalement les ressources dont ils ont besoin pour être compétitifs à l'échelle internationale, attirer des professeurs et des chercheurs de haut niveau et mettre en œuvre des politiques intégrées englobant la recherche, l'éducation et le transfert technologique.

\section{Une cohorte de population de référence en santé}

L'objectif global de l'étude Milieu Intérieur est donc d'évaluer les facteurs qui expliquent la variabilité immunologique au sein de la population générale saine. Pour cela, une étude clinique transversale sur la santé de la population appelée « ́́tude des déterminants génétiques et environnementaux de la variabilité de la réponse immunitaire : vers une médecine personnalisée ${ }^{2} \gg$ a été initiée. L'objectif premier de cette étude est de définir les facteurs génétiques et environnementaux qui contribuent à l'hétérogénéité des réponses immunitaires. II s'agit de caractériser et d'intégrer (1) les habitudes de vie quotidienne, au moyen d'un questionnaire détaillé ; (2) la

${ }^{2}$ Numéro d'enregistrement de la recherche (ID-RCB): 2012-A00238-35. 


\section{The Milieu Intérieur Project \\ Hosted by Institute Pasteur on behalf of the Milieu Intérieur Consortium}

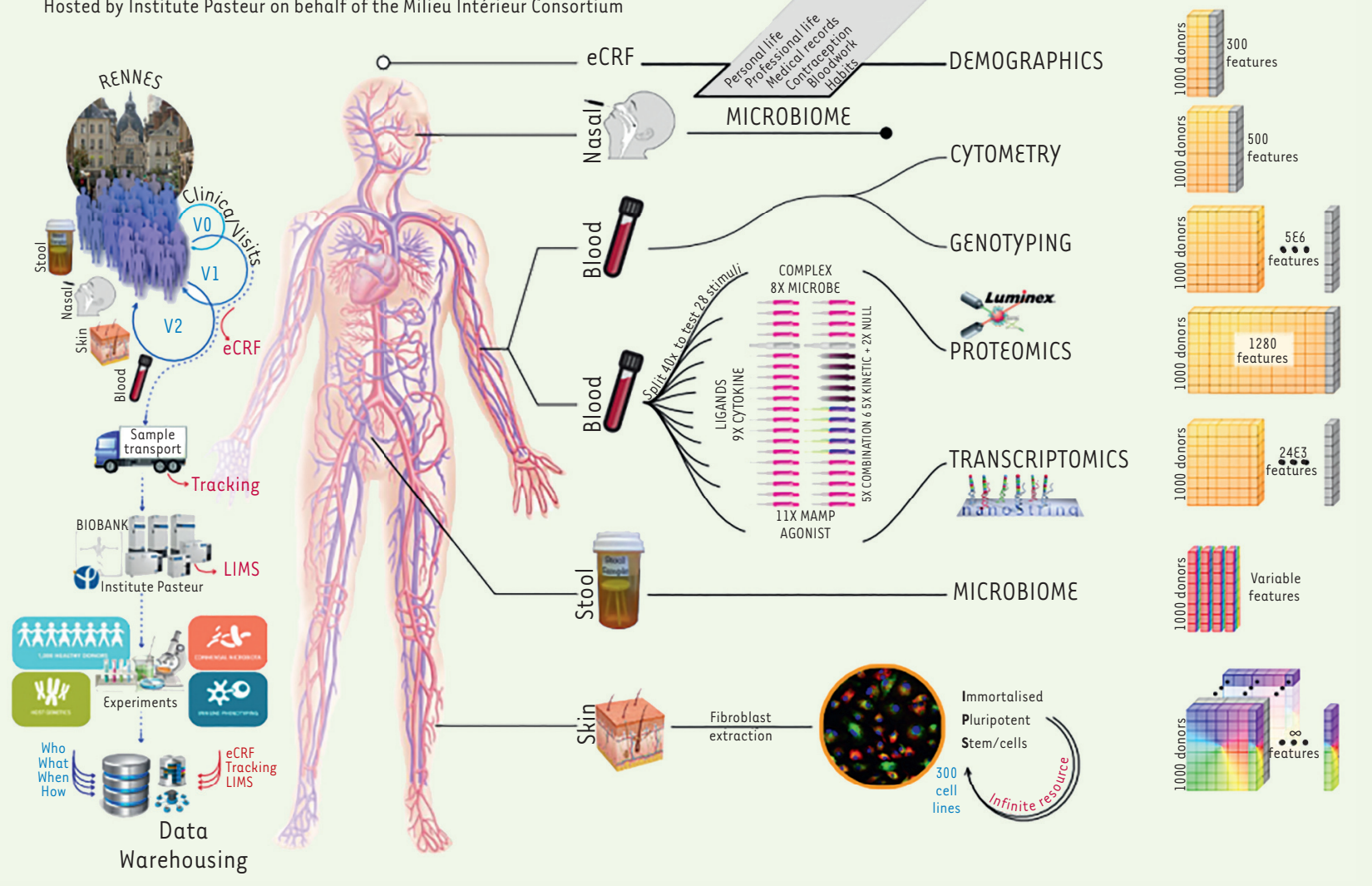

Figure 2. Illustration du processus de collecte et d'analyse des échantillons prélevés sur les 1000 donneurs sains de Milieu Intérieur dans le centre de recrutement de Rennes.

variabilité génomique, par le génotypage des SNP à l'échelle du génome et du séquençage de l'exome entier; (3) la diversité métagénomique fondée sur l'analyse séquentielle des populations bactériennes, fongiques et virales dans les échantillons fécaux et nasaux réalisés chez les individus; (4) les signatures transcriptionnelles et protéiques induites par des microbes entiers ou des agonistes microbiens (MAMP, microbeassociated molecular pattern) identifiés, des cytokines ou des stimulus spécifiques de la réponse lymphocytaire $T$; et (5) les niveaux circulants de populations de cellules immunitaires, par cytométrie en flux. Un autre objectif est d'établir une banque de cellules, dont des lignées de lymphocytes B transformées par le virus d'Epstein-Barr et des fibroblastes, à partir d'individus sains génétiquement annotés, afin de les utiliser dans des études mécanistiques. Pour atteindre ces objectifs, un total de 1000 volontaires en bonne santé, répartis également selon le sexe (sex-ratio 1:1) et stratifiés sur cinq décennies de vie, ont été recrutés.

\section{Définir la santé pour comprendre la maladie}

Afin de définir les paramètres caractéristiques d'une population en bonne santé, un groupe de travail, composé d'experts représentant différentes spécialités cliniques (biologie médicale, médecine régénérative, allergie, pédiatrie, nutrition, psychiatrie, médecine de laboratoire) et scientifiques (immunologie, microbiologie, génétique, épidémiologie, méthodologie, sociologie), a été formé pour établir les critères définissant un individu comme donneur «sain », tout en préservant la faisabilité du recrutement et permettant une analyse statistique robuste.

Ce groupe de travail a ainsi défini les critères généraux d'admissibilité pour présélectionner les sujets (âge, sexe, indice de masse corporelle, ascendance autodéclarée, lien de parenté avec les autres sujets). Des critères d'exclusion portant sur des caractères pouvant avoir une incidence sur le système immunitaire et/ou les procédures d'étude (comme des maladies chroniques, des sujets atteints de troubles cutanés qui pourraient compromettre une biopsie cutanée) ont été déterminés. Des facteurs médicaux, physiologiques et comportementaux ont également été retenus selon leurs conséquences possibles sur l'activité des cellules immunitaires ou le 
microbiote. La prévalence des caractéristiques des donneurs a été prise en compte, en excluant des phénotypes inférieurs à $1 \%$ de la population afin d'assurer une puissance suffisante pour les études d'association. Enfin, les critères trop conservateurs, sélectionnant une population « en très bonne santé », ont été écartés.

Afin de pouvoir associer la variation génétique et épigénétique à des phénotypes définis, une liste de critères d'inclusion et d'exclusion a été établie pour recruter des volontaires dont le système immunitaire est le moins perturbé : les donneurs ne devaient pas présenter de signes ou faire état d'antécédents de troubles neurologiques ou psychiatriques, ou d'états pathologiques graves, chroniques ou récurrents; des signes d'abus d'alcool, de consommation récente de drogues (y compris l'utilisation récente de cannabis), d'administration récente de vaccins et utilisation d'agents immunomodulateurs, d'exposition actuelle et antérieure à des traitements médicaux (aspirine) ou d'utilisation de produits alternatifs (homéopathie, huiles essentielles). La naissance prématurée a aussi été prise en compte. Pour éviter l'influence des fluctuations hormonales chez les femmes, seules les femmes préménopausées ou postménopausées ont été incluses. Afin d'éviter la présence de souspopulations dans la population étudiée, ce qui aurait une incidence sur la détection des associations génotype-phénotype, l'étude a été limitée aux personnes d'origine européenne, citoyens français métropolitains depuis trois générations au moins (les parents et les grands-parents des sujets doivent être nés également en France métropolitaine). Cette stratégie a été choisie en fonction de la nécessité d'avoir le maximum de puissance statistique pour l'analyse génétique, et donc de se concentrer sur une population génétiquement homogène. Conscients de cette limitation pour refléter la diversité de la population française en général, nous entreprenons actuellement des études similaires dans des populations saines d'origine africaine et asiatique.

Alors que certains facteurs d'exclusion étaient facilement applicables, comme les infections chroniques (hépatite $C$ par exemple) ou les maladies graves (cancer, auto-immunité, etc.), d'autres se sont avéré plus difficiles, comme le seuil à prendre en compte pour les allergies, l'exposition à des toxines connues (tabac) ou la présence de signes pré-syndromiques (hypertension). Bien que l'utilisation de valeurs de référence pour les paramètres hématologiques, biochimiques et sérologiques, communément acceptées en clinique pour définir la santé d'un individu, soit considérée comme un critère d'inclusion/exclusion, la perte potentielle de phénotypes extrêmes a été prise en compte avec le choix de valeurs seuils pouvant indiquer la nécessité d'un suivi médical (par exemple, des concentrations d'enzymes hépatiques supérieures à trois fois la limite supérieure de la normalité).

Finalement, les paramètres attendus chez plus de $5 \%$ des personnes échantillonnées ont été sélectionnés en excluant toute affection nécessitant un traitement médical passé ou actuel. Les antécédents médicaux personnels et familiaux ont été systématiquement consignés et les métadonnées associées ont été utilisées pour définir les associations génétiques, immunologiques et d'entérotypes et/ou pour faire régresser les facteurs pouvant potentiellement entraîner une confusion. Cet ensemble de critères pris en considération dans l'étude devrait aider la communauté internationale à conclure quant à une définition consen- suelle d'un état de bonne santé pour les études immunologiques lorsque des populations de donneurs en bonne santé, d'origine africaine et asiatique, auront également été étudiées.

La production de données génétiques (fondées sur le génotypage du génome et le séquençage de l'exome entier) et de phénotypes multiples (moléculaires, cellulaires et au niveau des organismes) dans les échantillons disponibles de la cohorte étudiée a généré un grand nombre de données permettant d'entreprendre des fouilles de ces dernières pour identifier des associations et accroître nos connaissances sur les différents facteurs impliqués dans la régulation des réponses immunitaires.

\section{Nécessité d'un suivi immunologique standardisé}

Bien que le phénotypage des populations cellulaires puisse révéler d'importants déterminants de la variabilité immunitaire, les réponses immunitaires sont très dynamiques et nécessitent souvent une stimulation ou une perturbation pour révéler toute leur profondeur et leur fonction. Par exemple, les études portant sur l'efficacité des vaccins exigent souvent une stimulation antigénique ex vivo des cellules du receveur afin d'évaluer par cytométrie en flux intracellulaire ou des tests de type ELISPOT, l'induction de l'immunité à médiation cellulaire T. De nombreux efforts ont récemment été réalisés afin de normaliser les procédures et les résultats obtenus avec ces approches [4].

Ces techniques reposent généralement sur l'isolement des cellules mononucléées du sang périphérique (PBMC) de l'individu. Cependant, cet isolement introduit une variabilité technique et reste difficile à normaliser au sein d'un même laboratoire et entre laboratoires. Le sang est, par exemple, souvent transporté des sites cliniques aux laboratoires centraux, ce qui peut entraîner une variabilité pré-analytique. La manipulation des cellules immunitaires innées peut également induire une activation non spécifique ou la mort des cellules prélevées. Pour limiter ces problèmes, des essais de stimulation sur sang total ont été développés (par utilisation de seringues TruCulture) dans le cadre de l'étude Milieu Intérieur. Néanmoins, contrairement aux PBMC isolés, le sang total comprend également des granulocytes, des plaquettes et des composants plasmatiques qui peuvent participer aux réponses immunitaires qui seront examinées. Nous avons décrit récemment une étude clinique multicentrique comparant les tests sur sang total (via TruCulture) aux tests conventionnels réalisés sur des PBMC isolés, en utilisant des lipopolysaccharides bactériens (LPS) et des anticorps combinés anti-CD3/CD28 pour stimuler la réponse cellulaire, l'activité des cellules étant révé- 
lée par la réactivité des surnageants de culture dans des essais sur protéines multi-analyses de type Luminex ${ }^{3}$ [5]. Le principal résultat en a été l'observation d'une meilleure reproductibilité, en particulier entre les centres, des tests TruCulture par rapport à la stimulation sur PBMC isolés, ce qui souligne la pertinence de cette approche pour les études multicentriques [5]. D'autres groupes ont utilisé des stimulations normalisées similaires à base de plaques de culture de sang total qui nécessitent des volumes sanguins plus faibles, ce qui constitue un avantage pour les études pédiatriques [6].

\section{Conclusion}

Plusieurs études récentes portant sur le phénotypage génétique et immunologique intégrés dans des populations saines ont commencé à définir les facteurs à l'origine des variations que l'on observe entre réactions immunitaires des individus [1]. II s'agit notamment de cohortes établies pour répondre à des questions similaires à celles posées dans le cadre de Milieu Intérieur : en particulier, le Human Functional Genomics Project [7], le Human Immunology Project Consortium (HIPC) [8], et le 10K Immunomes [9]. Collectivement, ces études et les premiers résultats obtenus dans le cadre de Milieu Intérieur ${ }^{4}$ apportent une meilleure définition de la variabilité de la réponse immunitaire et des facteurs clés qui la déterminent. Selon le phénotype immunitaire étudié, on estime ainsi que jusqu'à 50 \% de la variation observée proviennent de facteurs environnementaux [10], et entre 20 et $40 \%$ reposent sur la génétique [11-14]. Toutefois, il reste à définir un nombre important de facteurs individuels spécifiques à l'origine de cette variation, en particulier, les facteurs environnementaux spécifiques qui agissent sur l'immunité. Le pourcentage élevé de variation restant inexpliqué est probablement attribuable à des interactions complexes entre les gènes et l'environnement. Ceci nécessitera des études à grande échelle et longitudinales sur les populations pour que ces interactions soient décodées. Néanmoins, une telle compréhension est cruciale pour réaliser la promesse de stratégies de vaccination de précision qui tiendront compte de la réponse immunitaire spécifique d'un individu afin d'optimiser leur efficacité et de réduire leurs effets indésirables. Les stratégies de santé publique pourront alors tirer profit des progrès scientifiques récents et être adaptées pour relever les défis du XXI siècle, avec une population mondiale diversifiée sur les plans biologique, génétique et environnemental. $\diamond$

\section{SUMMARY}

Milieu Interieur: defining a healthy immune response for a better understanding of diseases

What all individuals have in common is that they are all different. Some of the greatest differences between any two individuals may be related to their immune responses. These differences may result in variable outcome to infection, disease severity and response to medical therapies. Differences in immune responses are partly resulting from evolutionary

${ }^{3}$ Une méthode permettant de définir plusieurs réponses à partir d'un échantillon unique.

${ }^{4}$ Ces résultats seront publiés dans un prochain numéro de médecine/sciences. forces acting at the level of host genetics and may also be due to differences encountered through environment and lifestyle. Despite these well-known inter-individual differences, this inherent variability is rarely included in clinical approaches or drug development. The LabEx Milieu Interieur consortium was established in 2011 to better define these immune differences in a healthy population and identify their genetic and environmental determinants. Such an understanding is crucial to achieve the promise of precision medicine that will take into account an individual specific immune response to ensure that public health strategies capitalize on recent scientific progress. $\diamond$

\section{REMERCIEMENTS}

Je remercie Julian Descles (Institut Pasteur) pour son aide à la traduction.

\section{LIENS D'INTÉRÊT}

L'auteur déclare n'avoir aucun lien d'intérêt concernant les données publiées dans cet article.

\section{RÉFÉRENCES}

1. Liston A, Carr $\varepsilon$ J, Linterman MA. Shaping variation in the human immune system. Trends Immunol 2016; 37 : 637-46.

2. McDermott AB, Cohen SB, Zuckerman JN, Madrigal JA. Hepatitis B thirdgeneration vaccines: improved response and conventional vaccine nonresponse : evidence for genetic basis in humans. J Viral Hepat 1998 ; 5 (suppl 2): 9-11

3. Thomas S, Rouilly V, Patin $\varepsilon$, et al. The Milieu Intérieur study : an integrative approach for study of human immunological variance. Clin Immunol 2015 ; $157: 277-93$.

4. Janetzki S, Britten CM, Kalos M, et al. MIATA-minimal information about $T$ cell assays. Immunity $2009 ; 31: 527-8$.

5. Duffy D, Rouilly V, Libri V, et al. Functional analysis via standardized wholeblood stimulation systems defines the boundaries of a healthy immune response to complex stimuli. Immunity 2014 ; $40: 436-50$.

6. Smolen KK, Ruck CE, Fortuno ES $3^{\text {rd }}$, et al. Pattern recognition receptormediated cytokine response in infants across 4 continents. J Allergy Clin Immunol 2014 ; 133 : 818-26.e4.

7. Pappalardo JL, Hafler DA. The human functional genomics project : understanding generation of diversity. Cell 2016 ; 167 : 894-6.

8. Brusic V, Gottardo R., Kleinstein SH, et al. Computational resources for high-dimensional immune analysis from the Human immunology project consortium. Nat Biotechnol 2014 ; 32 : 146-8.

9. Zalocusky KA, Kan MJ, Hu Z, et al. The 10,000 Immunomes project : building a resource for human immunology. Cell Rep 2018 ; 25 : 513-522.e3.

10. Carr EJ, Dooley J, Garcia-Perez JE, et al. The cellular composition of the human immune system is shaped by age and cohabitation. Nat Immunol $2016 ; 17: 461-8$.

11. Olaru ID, Lange $C$, Heyckendorf J. Personalized medicine for patients with MDR-TB. J Antimicrob Chemother 2016 ; 71 : 852-5.

12. Mangino M, Roederer M, Beddall MH, et al. Innate and adaptive immune traits are differentially affected by genetic and environmental factors. Nat Commun 2017 ; $8: 13850$.

13. Piasecka B, Duffy D, Urrutia A, et al. Distinctive roles of age, sex, and genetics in shaping transcriptional variation of human immune responses to microbial challenges. Proc Natl Acad Sci USA 2018 ; 115 : ع488-97.

14. Patin $\varepsilon$, Hasan M, Bergstedt J, et al. Natural variation in the parameters of innate immune cells is preferentially driven by genetic factors. Nat Immunol $2018 ; 19: 645$.

TIRÉS À PART

D. Duffy 